\title{
Blood and marrow transplantation during the emerging COVID-19 pandemic: the Seattle approach
}

\author{
Masumi Ueda Oshima $\mathbb{1}^{1,2,3} \cdot$ Brenda M. Sandmaier ${ }^{1,2,3} \cdot$ Effie Petersdorf ${ }^{1,2,3} \cdot$ Mary E. Flowers ${ }^{1,2,3}$. \\ Geoffrey R. Hill ${ }^{1,2,3} \cdot$ Stephanie J. Lee ${ }^{1,2,3} \cdot$ Frederick R. Appelbaum ${ }^{1,2,3} \cdot$ Paul A. Carpenter $^{1,2,4} \cdot$ K. Scott Baker ${ }^{1,2,4}$. \\ Laura Connelly-Smith ${ }^{1,2,5} \cdot{\text { Andrea } \mathrm{McCool}^{2} \cdot \text { Suni } \mathrm{Elgar}^{2} \cdot \text { Steven A. Pergam }}^{2,6,7} \cdot$ Catherine Liu $\mathbb{1}^{2,6,7}$. \\ F. Marc Stewart ${ }^{1,2,3} \cdot$ Marco Mielcarek $k^{1,2,3}$
}

Received: 21 July 2020 / Revised: 9 September 2020 / Accepted: 16 September 2020 / Published online: 26 September 2020

(c) The Author(s), under exclusive licence to Springer Nature Limited 2020

\begin{abstract}
On January 20, 2020, the first patient with coronavirus disease 2019 (COVID-19) in the United States of America was diagnosed in Washington state, which subsequently experienced rapidly increasing numbers of COVID-19 cases, hospitalizations, and deaths. This placed the Seattle Blood and Marrow Transplant Program at Fred Hutchinson Cancer Research Center (Fred Hutch) in the national epicenter of this pandemic. Here, we summarize the experience gained during our rapid response to the COVID-19 pandemic. Our efforts were aimed at safely performing urgent and potentially lifesaving stem cell transplants in the setting of pandemic-related stresses on healthcare resources and shelter-in-place public health measures. We describe the unique circumstances and challenges encountered, the current state of the program amidst evolving COVID-19 cases in our community, and the guiding principles for recovery. We also estimate the collateral impact of directing clinical resources toward COVID-19-related care on cancer patients in need of stem cell transplantation. Although our experience was influenced by specific regional and institutional factors, it may help inform how transplant programs respond to COVID-19 and future pandemics.
\end{abstract}

\section{Introduction}

The first confirmed case of coronavirus disease 2019 (COVID-19) caused by the severe acute respiratory

Masumi Ueda Oshima

mueda@fredhutch.org

1 Clinical Research Division, Fred Hutchinson Cancer Research Center, Seattle, WA, USA

2 Seattle Cancer Care Alliance, Seattle, WA, USA

3 Division of Medical Oncology, Department of Medicine, University of Washington, Seattle, WA, USA

4 Department of Pediatrics, University of Washington, Seattle, WA, USA

5 Division of Hematology, Department of Medicine, University of Washington, Seattle, WA, USA

6 Vaccine and Infectious Diseases Division, Fred Hutchinson Cancer Research Center, Seattle, WA, USA

7 Division of Allergy and Infectious Diseases, Department of Medicine, University of Washington, Seattle, WA, USA syndrome coronavirus 2 (SARS-CoV-2) in the United States of America was reported on January 20, 2020, in Snohomish County, Washington state [1]. As of July 18, 2020, Washington state had reported almost 47,000 cases of and more than 1400 deaths related to COVID-19 [2]. Situated in the initial epicenter of the pandemic, the Blood and Marrow Transplant (BMT) Program at Fred Hutchinson Cancer Research Center (Fred Hutch)/Seattle Cancer Care Alliance (SCCA) was at the forefront of delivering care to transplant patients during the surge of this public health crisis.

While $~ 50 \%$ of our referral base originates from King County (where Seattle is located) and the surrounding counties of Pierce and Snohomish, our catchment area includes Washington, Wyoming, Alaska, Montana, and Idaho. King County accounts for $\sim 30 \%$ of Washington state's population of 7.6 million, but as of July 18, 2020, it had $38 \%$ of COVID-19-related hospitalizations and $44 \%$ of COVID-19-related deaths [2] (Fig. 1). With the rapid rise of COVID-19 cases in Washington state and particularly in the King County metropolitan area in early March 2020, our BMT program immediately implemented 
Fig. 1 COVID-19 activity in Washington state and King County. The Fred Hutchinson Cancer Research Center/Seattle Cancer Care Alliance are located in north-western King County. The frequency of confirmed COVID-19 cases in Washington state according to county is shown on the map. The inserted table list confirmed cases, hospitalizations, deaths, and case-fatality rates related to COVID-19 in Washington state and King County. This information was updated on July 18,2020 . The map was adapted from data provided by the Washington State Department of Health (doh.wa.gov).

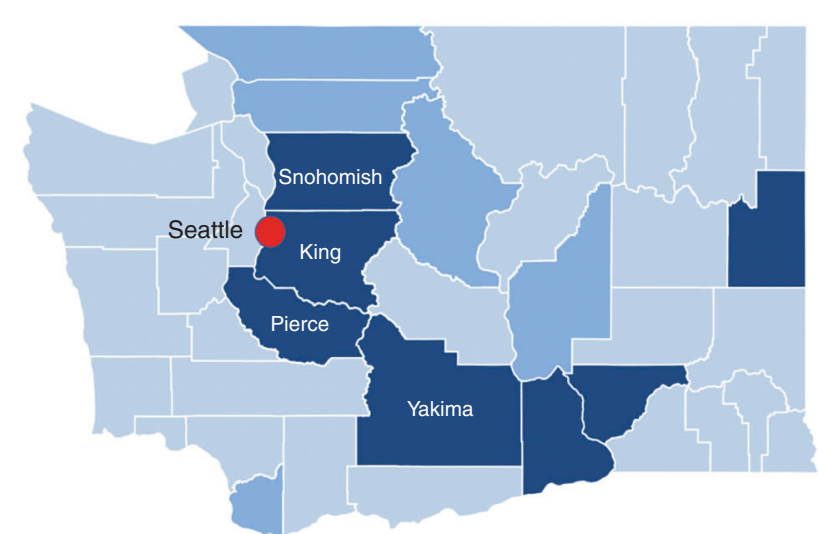

Legend

$2,640+$

1,981 to 2,640

1,321 to 1,980

661 to 1,320

1 to 660

0

\begin{tabular}{|l|c|c|c|}
\hline & Washington state & King county & Proportion of WA state \\
\hline Population & 7.6 Mio & 2.25 Mio & $30 \%$ \\
\hline Confirmed cases & 46,946 & 13,153 & $28 \%$ \\
\hline Hospitalizations & 5,063 & 1,925 & $38 \%$ \\
\hline Deaths & 1,447 & 635 & $44 \%$ \\
\hline Case fatality ratio & $3.1 \%$ & $4.8 \%$ & N/A \\
\hline
\end{tabular}

several changes (Fig. 2). Over the ensuing 10 weeks, we prepared for worst-case scenarios with predicted substantial shortages of personal protective equipment, acute and intensive care hospital beds, clinical staff and other vital resources as the number of COVID-19-positive cases rapidly increased. The key components of our BMT program's response to the COVID-19 pandemic are summarized in Table 1.

Until a vaccine becomes available, herd immunity is established, or effective treatments are developed, the evolution of the COVID-19 pandemic remains uncertain [3, 4]. Depending on the region in the United States of America, there are concerns for insufficient control of the current wave of infections and future waves, which may require reinstitution of some of the mitigation strategies taken initially during the first few weeks of the pandemic. As a large transplant center operating throughout this public health emergency, we have learned much about continuing to provide care to transplant patients in need of timely treatment. Here, we share our preliminary experience, lessons learned, preparation for recovery, and continued response to the evolving pandemic.

\section{Initial rapid response}

From the earliest days of the pandemic, our program's response was closely aligned with that of our clinical partner organizations that comprise the SCCA: Fred Hutch, University of Washington (UW), and Seattle Children's Hospital, with representation of program leadership on the SCCA incident command structure [5]. We were fortunate to have early access to robust SARS-CoV-2 testing developed and performed at UW Laboratory Medicine. In addition, infection prevention measures were rapidly upscaled, including an institution-wide work-from-home mandate for nonessential staff, point-of-entry symptom screening of all staff, patients and visitors at our outpatient clinics and hospital, and SARS-CoV-2 PCR testing of patients and staff with respiratory symptoms. Concurrent with practice across the UW hospitals and clinics, the BMT program deferred all nonurgent procedures and transplants (discussed below). In addition, our BMT program postponed referrals from outside of King and surrounding counties if transplantation was deemed less urgent. The UW hospital system rapidly implemented a no-visitor policy to minimize potential COVID-19 exposure of hospitalized patients and staff. The Seattle Children's Hospital limited patients to one caregiver/visitor. Patients coming to the outpatient clinic were restricted to one caregiver. We also rapidly implemented pretransplant SARS-CoV-2 testing of all patients to identify asymptomatic carriers who could have an increased risk of transplant-related complications and mortality, as well as to mitigate risk of transmission from asymptomatic individuals to other patients and staff. Lastly, in line with the UW hospitals and clinics, we promptly implemented telehealth visits in lieu of face-to-face encounters with patients referred for transplant consultation. This allowed us to establish and maintain essential communication with patients and referring oncologists yet minimize COVID-19 exposure during the height of the response.

\section{Operational changes}

After the initial rapid response, we focused on ensuring an adequate infrastructure to maintain operations during a time of rapid change. We convened a biweekly BMT COVID-19 


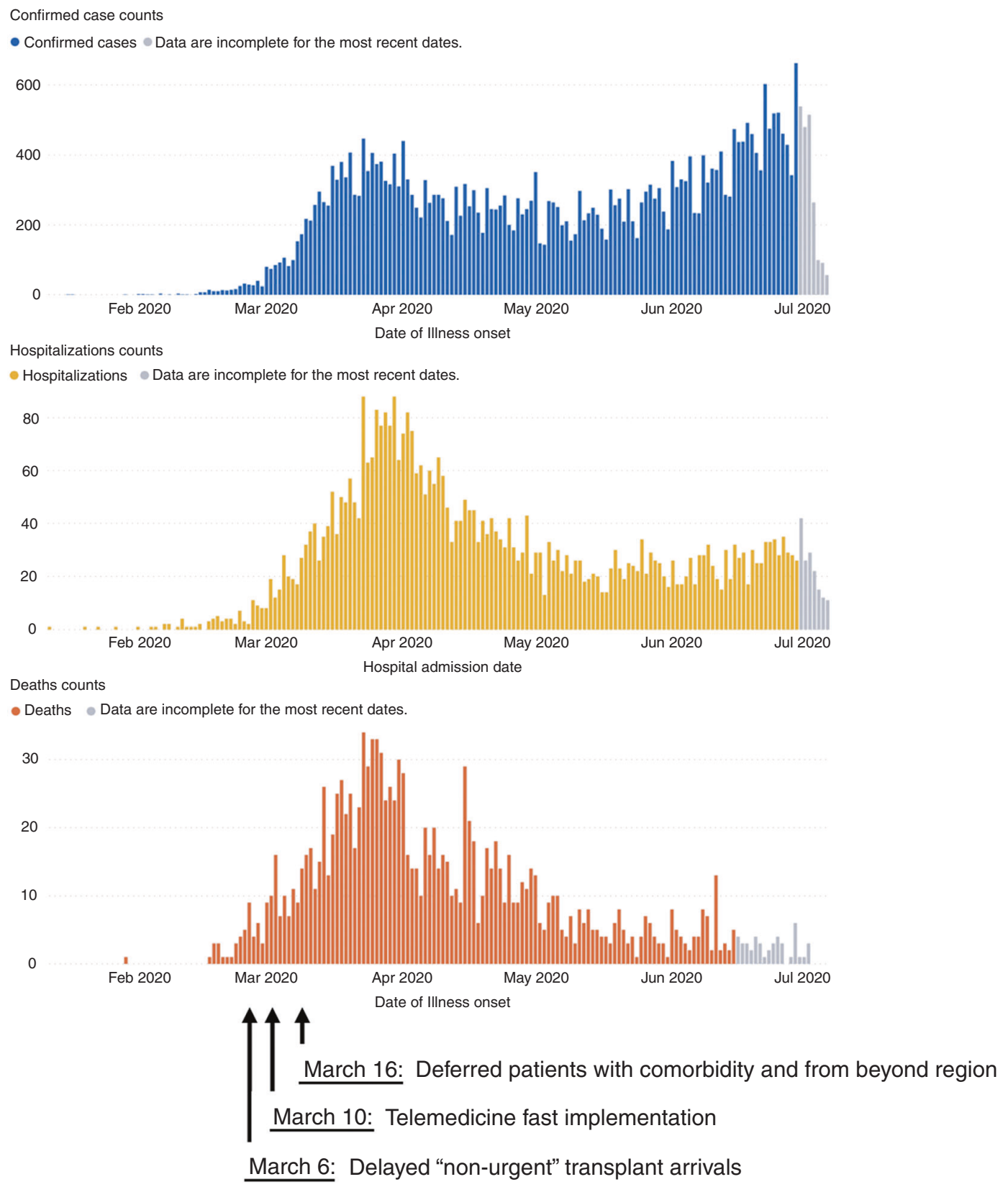

Fig. 2 Timeline of arrival restrictions for patients scheduled for transplant at the Fred Hutchinson Cancer Research Center/Seattle Cancer Care Alliance according to increasing COVID-19 activity in Washington state. Top panel: confirmed daily case counts; middle

committee videoconference with representation from infectious disease experts and nursing, physician, and administrative leaders to discuss all practice changes. A global approach for tracking and integrating information from local, national, and international resources was taken to develop strategies based on the most up-to-date data available. Operational changes were distributed to our faculty by regularly updated internal memoranda and faculty meetings. Letters to patients and referring oncologists explained the key changes to clinical practice and their rationale. panel: daily hospitalizations; and lower panel: daily mortality. This information was updated on July 7, 2020. The graphs were adapted from data provided by the Washington State Department of Health (doh.wa.gov) [2].

To ensure adequate physician staffing on the BMT service in case of absence due to illness or exposure, we established additional layers of back-up coverage. Physicians above the age of 65 years or those with relevant comorbidities were reassigned from clinic and hospital obligations to the telehealth consultation service. Similar arrangements were made for other clinical staff. With decrease in numbers of patients in the clinic, outpatient transplant nurses were redeployed for COVID-19 screening and triage services for the institution. 
Table 1 Key components of the BMT program's response to the COVID-19 pandemic.

\begin{tabular}{|c|c|}
\hline Category of response & Key steps \\
\hline Initial rapid response & $\begin{array}{l}\text { - Incident command structure established [5] } \\
\text { - SARS-CoV-2 PCR testing capacity secured } \\
\text { - Point-of entry screening at clinical facilities established } \\
\text { - Nonurgent and out-of-region transplants deferred } \\
\text { - Hospital no-visitor policy implemented } \\
\text { - Telehealth transplant consultations replaced face-to-face visits } \\
\text { - Work-from-home policy for nonessential staff implemented }\end{array}$ \\
\hline Operational changes & $\begin{array}{l}\text { - Additional inpatient rooms converted to negative pressure } \\
\text { - Clinic and triage hours extended to preserve inpatient resources } \\
\text { - Videoconference rounding as feasible } \\
\text { - Additional back-up physician coverage secured } \\
\text { - Staff deemed at higher risk of COVID-19 morbidity reassigned } \\
\text { - Biweekly videoconferences with program leadership } \\
\text { - Internal memoranda to faculty regularly updated and distributed }\end{array}$ \\
\hline $\begin{array}{l}\text { SARS-CoV-2 } \\
\text { testing by PCR }\end{array}$ & $\begin{array}{l}\text { - All symptomatic patients } \\
\text { - Asymptomatic patients: } \\
\text { - At the start of the pretransplant evaluation } \\
\text { - Before initiating the preparative regimen } \\
\text { - Weekly after HCT until discharge to local physician } \\
\text { - Prior to any procedure }\end{array}$ \\
\hline Prioritization of transplants & $\begin{array}{l}\text { - Considered risks related to the underlying malignancy (Table 2) and } \\
\text { comorbidity } \\
\text { - Prioritized local over out-of-region patients } \\
\text { - Generally proceeded with HCT if long-term survival estimate }>20 \%\end{array}$ \\
\hline Donor considerations & $\begin{array}{l}\text { - Unrelated donors: cryopreserve all products } \\
\text { - Related donors: negative SARS-CoV-2 testing within } 48 \mathrm{~h} \text { of start of } \\
\text { patient conditioning permitted use of fresh donor product }\end{array}$ \\
\hline Clinical trials & $\begin{array}{l}\text { - Temporary pause of all phase } 1 \text { and } 3 \text { trials } \\
\text { - Phase } 2 \text { trials with potential benefit to patients continued accrual } \\
\text { - Study visits conducted by telehealth } \\
\text { - Ancillary studies paused }\end{array}$ \\
\hline
\end{tabular}

On the 40-bed inpatient BMT unit, eight negative pressure rooms in addition to the existing four were created by installing mobile pressure units in standard (positive pressure) rooms to prepare for specialized care of transplant patients infected with COVID-19. In order to reduce emergency room utilization, an outpatient acute care clinic for both transplant and nontransplant oncology patients was established. Extended infusion clinic hours (from 7 a.m. to 10 p.m.) allowed outpatient administration of regimens that would otherwise have necessitated inpatient admission for prolonged hydration or toxicity monitoring.

To maximize physical distancing in outpatient clinics and on inpatient units, rounding of multi-disciplinary teams was modified by leveraging video conferencing for discussion of patients before in-person rounding. After verifying that an adequate supply of masks would be available, we implemented universal masking of all patients, visitors, and staff. Finally, as the Seattle Children's Hospital had a significantly lower caseload of COVID-19 patients compared to the adult medicine services at UW, any willing adolescent and young adult patient in need of urgent transplantation was transferred to receive care by our pediatric BMT colleagues.

\section{SARS-CoV-2 testing}

In early March 2020 when community transmissions resulted in a steep increase of COVID-19 cases in Washington state, the individual risk for hematopoietic cell transplant (HCT) patients who acquired the infection was unknown. We extrapolated the experience from other respiratory viral infections where, compared to immunecompetent individuals, lower respiratory tract involvement after allogeneic (and to a lesser extent autologous) transplantation is more common during the peritransplant period with profound immunosuppression. Our goal was therefore to defer HCT until patients were asymptomatic and no longer shedding virus. For asymptomatic carriers, the goal was to document a negative PCR from a nasopharyngeal swab before proceeding to HCT. Monitoring the prevalence of COVID-19 among our transplant population was also important to ensure the safety of other patients and staff. Therefore, patients were tested with nasopharyngeal or anterior nares SARS-CoV-2 PCR as follows: (i) when presenting with symptoms consistent with COVID-19; (ii) all asymptomatic patients upon entering our system at the start of the pretransplant evaluation and before initiating the 
preparative regimen; (iii) weekly after HCT until referral to local oncologists; and (iv) prior to any procedure. Between March 6 and July 18, 1314 tests in 259 unique transplant patients had been performed without returning a single confirmed positive result. This compared to the following positive test results (COVID-19 antigen detection by PCR): Washington state residents, 5.8\% $(N=809,339)$; cancer patients at SCCA, $2.7 \%(N=2358)$; and SCCA staff, $1.8 \%$ $(N=1182)$. Although testing of asymptomatic caregivers and family members was considered, it was not implemented due to the logistical challenges of establishing testing for individuals not directly under our care. Routine testing of asymptomatic staff, the utility of which likely depends on community prevalence of COVID-19, was not performed.

\section{Prioritization of transplants}

In early March 2020, the Institute for Health Metrics and Evaluation at the UW predicted that the COVID-19-driven demand for healthcare resources including acute and intensive care hospital beds and ventilators might exceed the available supply in Washington state and particularly in our region [6]. Transplant patients requiring inpatient care typically occupy inpatient beds for a prolonged period and often require higher levels of care such as intensive care units, which are resources that must be shared with the nontransplant patient population. Our program was thus confronted with the unprecedented challenge of balancing our ability to safely perform potentially life-saving HCT against the prospect that locally available and vital healthcare resources might soon get exhausted. We therefore devised an algorithm to help guide the temporary deferral of transplants deemed nonurgent based on the risk of the underlying malignancy (Table 2). At the same time, we prioritized patients for transplant who had the highest anticipated benefit ( $>20 \%$ expected rates of longer-term survival) from immediate transplantation based on both the risks of recurrence or progression of the malignancy and burden of comorbidity. Patients with preexisting significant cardiopulmonary comorbidities were deferred because the potential morbidity and mortality associated with posttransplant COVID-19 infection was thought to outweigh the expected benefit of transplantation. Stem cell mobilization and collection for autologous transplant patients continued, but transplant was deferred in most cases. Transplantation for patients with nonmalignant conditions was generally deferred. In rare situations, mostly for pediatric immunodeficiency disorders, discussion with local disease experts determined whether the risk-to-benefit ratio of delaying transplant was acceptable. Many patients already undergoing pretransplant evaluations were discharged back to their local oncologists to receive maintenance chemotherapy in anticipation of later 


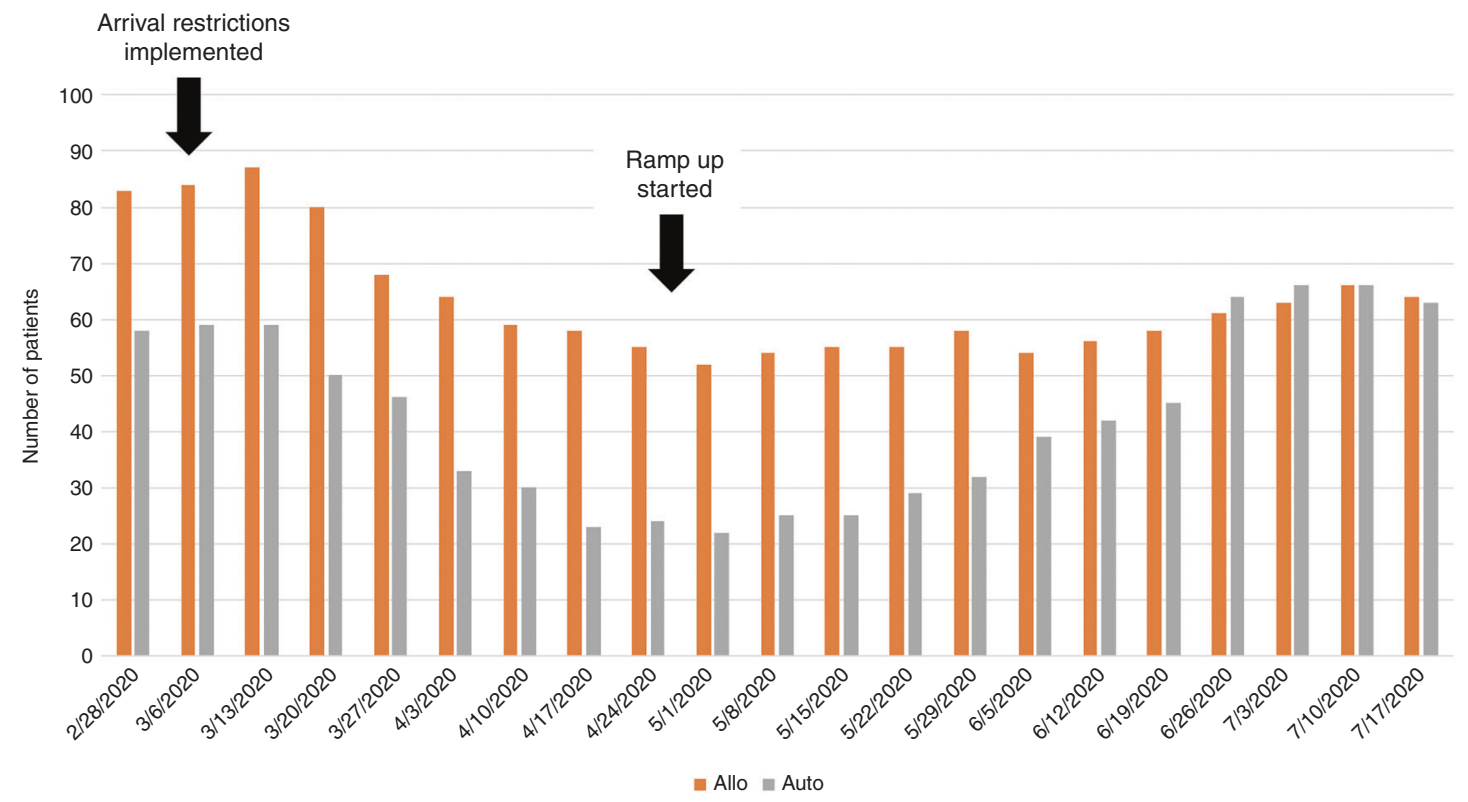

Fig. 3 Impact of COVID-19-related arrival restrictions on patient census. Effective March 6, 2020, only higher urgency patients were accepted for evaluation and transplantation at the Fred Hutchinson Cancer Research Center/Seattle Cancer Care Alliance (see also

transplant dates. All deferrals were carefully coordinated with referring providers to assure that an individualized interim care plan for each patient was in place.

To reduce utilization of hospital resources, additional consideration was given to the types of preparative regimens used. For example, unless evidence-based outcomes data indicated a benefit of myeloablative over nonmyeloablative or reduced-intensity conditioning, the lowerintensity approach was chosen. No specific graft-versushost disease (GVHD) prophylaxis strategy was preferred.

With the above outlined approach to prioritizing transplants, during the peak of the pandemic in our region from March 6 until May 31 (Figs. 2 and 3), 15 of 55 (27\%) planned allogeneic and 31 of $53(58 \%)$ planned autologous transplant patients were redirected to continuing nontransplant oncology care (total 46 of 108 patients [43\%] were deferred). Thus, for a period of $\sim 3$ months, transplants deemed nonurgent or transplants in patients with significant comorbidities were delayed. As of July 10, 2020, 1 month following the phased ramp up of our BMT program in response to declining COVID-19 activity in the community (discussed below), 25 of the 46 deferred patients (54\%) have been rescheduled for transplant. In the future, we hope to assess how often transplant delays might have led to a permanent loss of transplant opportunity or decreased chances of successful transplantation. This knowledge will help us prepare for a second wave of COVID-19 or other potential future public health crises that require rationing of critical healthcare resources.
Table 2). Data are shown according to allogeneic and autologous transplant patients under our program's care. As indicated, phase 1 of the ramp up was initiated on April 27, 2020.

\section{Donor considerations}

COVID-19 infection of the stem cell donor has potential implications for both the donor and the recipient. Infection of donors would make them ineligible for the donation process, either due to severity of symptoms or the unknown potential for viral transmission through the blood. Furthermore, as COVID-19 may be associated with a severe systemic inflammatory syndrome and hypercoagulation $[4,7-$ 10], the use of G-CSF even in asymptomatic COVID-19 antigen-positive donors raises concern for donor safety. Also, as international flight restrictions and other COVID19-related transport challenges may delay timely delivery of fresh allogeneic stem cell products, the National Marrow Donor Program (NMDP) required on March 30, 2020 that all unrelated donor cell products be cryopreserved prior to initiation of the recipients' conditioning regimen [11]. Based on local institutional policy, we began to also cryopreserve all related donor stem cell products due to concern that donors might encounter travel impediments or acquire COVID-19 immediately before or during stem cell mobilization. On a case by case basis, related donor stem cell products were collected at NMDP collection sites to eliminate the risk associated with the donor travel, in particular for donors traveling from a location with a relatively low prevalence of COVID-19 to our higher prevalence area. HLA-haploidentical related donors were sometimes prioritized over HLA-matched unrelated donors if the only product available was international. 


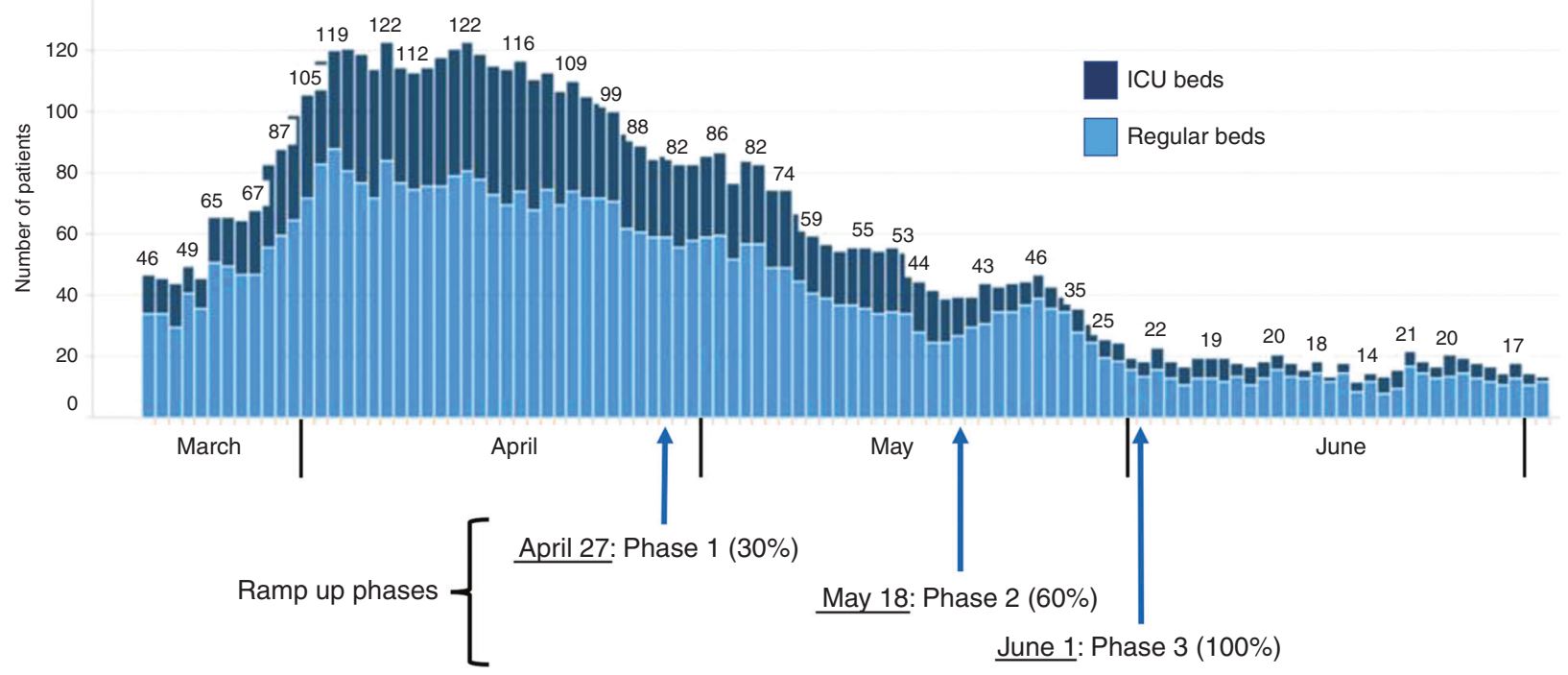

Fig. 4 Phased ramp up of hematopoietic cell transplantation activity after the peak of COVID-19-related hospitalizations in the University of Washington hospital system. Effective April 27, 2020, the first phase of a measured ramp up (30\% of new transplant patient arrivals compared to typical arrival volumes) was initiated. Phases 2 and 3 (60 and $100 \%$ of typical arrival volumes) were initiated on May

As the incidence of new COVID-19 cases continues to fluctuate in our local community, this policy continues to evolve. While we initially did not require universal SARSCoV-2 testing of asymptomatic related donors prior to stem cell mobilization or donation, we transitioned to testing all related donors within $48 \mathrm{~h}$ of the recipient's start of conditioning and transplant noncryopreserved product provided the tests are negative. However, to minimize COVID-19 exposure of donors, they must remain in the area and refrain from travel between the time of testing and start of stem cell mobilization or marrow donation.

\section{Clinical trials}

A large proportion of our patients are typically treated on clinical trials. To minimize utilization of clinical resources and unnecessary exposure of research support staff, enrollment on many clinical trials was paused during the surge of COVID-19 cases. Clinical trials without a demonstrable potential benefit for patients, such as phase 1 trials, were paused. Phase 2 clinical trials deemed potentially beneficial to patients continued to enroll. Phase 3 trials comparing an investigational arm to a well-defined standard-of-care alternative were paused. Patients already enrolled continued protocol-directed therapy, but whenever possible follow-up visits were done via telehealth. Nearly all national cooperative studies were closed to enrollment. Ancillary studies involving research blood draws were also paused in order to minimize requirements for research staff to be on campus. With the recent relaxation of shelter-in-
18 and June 1, respectively, as indicated. The graph shows the daily number of patients hospitalized (intensive care unit [ICU] beds and regular beds) with COVID-19 in the University of Washington hospital system, which provides inpatient care for Fred Hutchinson Cancer Research Center/Seattle Cancer Care Alliance hematopoietic cell transplant patients.

place recommendations for King County, trials have been reopened in a phased approach and synchronized with overall advancing clinical operations. Priority was given to trials deemed to have the greatest potential benefit for patients and those that were federally funded.

\section{Long-Term Follow-Up (LTFU) Program during COVID-19}

The Fred Hutch/SCCA LTFU clinical service offers lifelong consultations to about 6000 posttransplant patients and to their primary care providers via telephone and on-site evaluations. During the upswing and peak of COVID-19 hospitalizations at the UW (Fig. 4), routine annual LTFU evaluations were deferred. Nonurgent chronic GVHD evaluations were performed via telehealth visits. Exceptions to this approach were patients with progressive cutaneous sclerotic forms of chronic GVHD who could only be assessed in person and patients whose deferral beyond a few weeks was considered to have an unacceptably high risk of GVHD-related morbidity or mortality. LTFU telehealth visits allowed some evaluation of chronic GVHD including review of systems, rashes, skin ulcers, range of motion and evaluation of oral mucosa, and edema depending on the quality of patients' visual equipment. By June 2020, with decrease in COVID-19 cases in the community, in-person visits for GVHD consultations and comprehensive annual assessments resumed in a scaled manner. Telehealth visits remain an option for LTFU patients unable to return to our center. 


\section{Global collaboration}

The COVID-19 pandemic has highlighted the importance of dialog among transplant centers across the United States of America and worldwide to share best practices and guidelines. Already, collaboration across organizations such as the European Society for Blood and Marrow Transplantation (EBMT), American Society of Transplantation and Cellular Therapy (ASTCT), Center for International Blood and Marrow Transplant Research, NMDP, American Society of Hematology, and the Worldwide Network for Blood and Marrow Transplantation have advanced our understanding and preparations during the pandemic. Guidelines published by EBMT [12] and ASTCT [13] provide a framework for individual transplant center's response. Our institution's COVID-19 measures pertaining to pre-HCT evaluation, deferral of HCT candidates who test positive for SARS-CoV-2, procedures for HCT donors, and treatment considerations closely mirror those proposed by both ASTCT and EBMT. Slight variations reflect differences in regional epidemiology as well as resource availability. For example, our institution's conduct of weekly SARS-CoV-2 testing of all asymptomatic patients posttransplant is unique and is facilitated by the wide availability of testing at our institution [14]. These measures will need to be continually reviewed as the local epidemiology changes, and this approach may not be suitable for all transplant centers.

\section{Road to recovery}

As the rates of new COVID-19 infections, hospitalizations, and deaths in our community began to plateau and eventually decrease in mid-April $[2,6]$, we conceptualized a phased approach to gradually and safely ramp up transplant operations (Fig. 4). The following guiding principles informed the sequential approach to scheduling or rescheduling patients for transplant: patients who (i) had the highest expected HCT benefit-risk ratio; (ii) had previously arrived for evaluation but were deferred because of the COVID-19 surge; and/or (iii) were residents of King County and neighboring counties. The rationale for prioritizing regional patients was to enable patients to reside in their private residences to reduce the risk of exposure to COVID-19 in group housing, and avoid patients traveling from low-prevalence regions into our higher prevalence region. Finally, with input from institutional disease experts, we prioritized patient arrivals based on the perceived risk of delaying transplant according to underlying disease characteristics (Table 2). As future surges in COVID-19 activity occur, we envision taking a similar phased approach to scale down our transplant numbers.

\section{Future considerations}

The COVID-19 pandemic has confronted us and other transplant centers with unprecedented challenges with respect to providing timely and potentially life-saving HCT. As our region and the global community begin to recover from the first wave of COVID-19, we must stay vigilant as local and national epidemiology change. Many questions and uncertainties remain. It is unclear, for example, if and when transplantation activity around the world will return to pre-pandemic levels. Furthermore, the long-term impact of COVID-19-related transplant deferrals on patient outcomes, research program objectives, and the financial health of the institution require longitudinal assessment. At the same time, several operational changes necessitated by this pandemic, such as the rapid adoption of telehealth services, will likely remain vital components of care delivery models. As SARS-CoV-2 testing capacity and the local-regional prevalence of infection continues to fluctuate, the frequency of optimal testing of transplant recipients, donors, and caregivers has yet to be determined. Once a COVID-19 vaccine is available, vaccination approaches for the posttransplant period in immunosuppressed patients, vaccination of caregivers, family, and clinical staff, and even assessing potential benefits of vaccinating donors prior to stem cell donation will have to be determined. In light of this rapidly evolving body of knowledge, partnership with our professional organizations will be vital to advance our field, modify our approaches for other major respiratory viral pathogens, and improve outcomes for our patients despite the unprecedented challenges posed by this pandemic.

Acknowledgements The authors would like to thank all staff on the front lines at the Seattle Cancer Care Alliance, Fred Hutch, Seattle Children's Hospital, and the University of Washington Medical Center (UWMC) who have worked swiftly and tirelessly to ensure the safe care of transplant patients during the COVID-19 pandemic. We would especially like to thank all the staff in the Fred Hutch Clinical Coordinator's (Intake) Office, Unrelated Donor Transplant Program, Clinical Therapeutics Lab, Clinical Immunogenetic Laboratory, and SCCA Hospital/UWMC inpatient nurse managers and Advanced Practice Providers who were at the forefront of our BMT program's response to COVID-19. We would also like to thank our colleagues in the BMT program, Infectious Disease and Infection Prevention, Hematologic Malignancies, Pulmonary and Critical Care, Radiation Oncology, Nutrition, Pharmacy, Transfusion Medicine, Laboratory Medicine, Pathology, Palliative Care and Social Work who continue to support transplant patients during COVID-19. We also thank Dr. John Lynch for providing the data on COVID-19 hospitalizations rates at UWMC.

\section{Compliance with ethical standards}

Conflict of interest SAP receives research support from Global Life Technologies, Inc., and participates in research trials with Chimerix, Inc and Merck \& Co. He also currently participates in a clinical trial sponsored by the National Institute of Allergy and Infectious Diseases 
(U01-AI132004); vaccines for this trial are provided by Sanofi-Aventis. All other coauthors have no relevant competing interests to disclose.

Publisher's note Springer Nature remains neutral with regard to jurisdictional claims in published maps and institutional affiliations.

\section{References}

1. Holshue ML, DeBolt C, Lindquist S, Lofy KH, Wiesman J, Bruce $\mathrm{H}$, et al. First case of 2019 novel coronavirus in the United States. N Engl J Med. 2020;382:929-36.

2. Nieto Y, Patton N, Hawkins T, Spearing R, Bearman SI, Jones $\mathrm{RB}$, et al. Tacrolimus and mycophenolate mofetil after nonmyeloablative matched-sibling donor allogeneic stem-cell transplantations conditioned with fludarabine and low-dose total body irradiation. Biol Blood Marrow Transplant. 2006;12:217-25.

3. Moore KA, Lipsitch M, Barry JM, Osterholm, MT. COVID-19: the CIDRAP viewpoint. Part 1: the future of the COVID-19 pandemic: lessons learned from pandemic influenza. University of Minnesota: Minneapolis, Minnesota. Retrieved from https://www. cidrap.umn.edu/sites/default/files/public/downloads/cidrapcovid19-viewpoint-part1_0.pdf. Accessed Date: 8 Jul 2020.

4. Wiersinga WJ, Rhodes A, Cheng AC, Peacock SJ, Prescott HC. Pathophysiology, Transmission, Diagnosis, and Treatment of Coronavirus Disease 2019 (COVID-19): A Review. JAMA. 2020;324:782-93.

5. Ueda M, Martins R, Hendrie PC, McDonnell T, Crews JR, Wong TL, et al. Managing Cancer Care During the COVID-19 Pandemic: Agility and Collaboration Toward a Common Goal. J Natl Compr Cancer Netw. 2020;18:366-9.

6. The Institute for Health Metrics and Evaluation COVID-19 Projections. Retrieved from https://covid19.healthdata.org/united-sta tes-of-america. Accessed Date: 9 Jul 2020.
7. Klok FA, Kruip M, van der Meer NJM, Arbous MS, Gommers D, Kant KM, et al. Incidence of thrombotic complications in critically ill ICU patients with COVID-19. Thromb Res. 2020;191:145-7.

8. Spiezia L, Boscolo A, Poletto F, Cerruti L, Tiberio I, Campello E, et al. COVID-19-related severe hypercoagulability in patients admitted to intensive care unit for acute respiratory failure. Thromb Haemost. 2020;120:998-1000.

9. Connors JM, Levy JH. COVID-19 and its implications for thrombosis and anticoagulation. Blood. 2020;135:2033-40.

10. Mehta P, McAuley DF, Brown M, Sanchez E, Tattersall RS, Manson JJ, et al. COVID-19: consider cytokine storm syndromes and immunosuppression. Lancet. 2020;395:1033-4.

11. National Marrow Donor Program/Be The Match Network Announcement: New Transplant Center Requirement for Unrelated Donor Products - Newsletter. recieved 2020.

12. Ljungman, P., Mikulska, M., de la Camara, R. et al. The challenge of COVID-19 and hematopoietic cell transplantation; EBMT recommendations for management of hematopoietic cell transplant recipients, their donors, and patients undergoing CAR T-cell therapy. Bone Marrow Transplant. 2020:1-6. https://doi.org/10. 1038/s41409-020-0919-0. Epub ahead of print.

13. Waghmare A, Abidi M, Boeckh M, Chemaly RF, Dadwal SS, El Boghdadly Z, Papanicolaou GA, Pergam S, Shahid Z. Interim Guidelines for COVID-19 Management in Hematopoietic Cell Transplant and Cellular Therapy Patients, Version 1.3. 2020. Retrieved from. https://www.astct.org/viewdocument/a stct-interim-patient-guidelines-ap?CommunityKey $=\mathrm{d} 3949 \mathrm{~d} 84$ 3440-45f4-8142-90ea05adb0e5\&tab=librarydocuments. Accessed Date: 30 Jun 2020.

14. Lieberman JA, Mays JA, Wells C, Cent A, Bell D, Bankson DD, Greninger AL, Jerome KR, Limaye AP. Expedited SARS-CoV-2 screening of donors and recipients supports continued solid organ transplantation. Am J Transplant. 2020. https://doi.org/10.1111/ ajt.16081. Epub ahead of print. 